\title{
The crucial role of theoretical scholarship for learning design and technology
}

\author{
Richard E. West ${ }^{1} \cdot$ Peg Ertmer $^{2} \cdot$ Susan McKenney ${ }^{3}$
}

Published online: 25 April 2020

(c) The Author(s) 2020

The field of learning design and technology is unique among academic disciplines for various reasons. First, our field aims to accomplish both research and practical goals. In short, our discipline exists equally in both the worlds of design and practice, and in research and scholarship. Undergirding and driving our work in both of these areas is good theory. Solid theoretical foundations about learning, teaching, design, and technology separate instructional designers from website developers, teachers from presenters, and academics from commentators.

In addition, our field is a meta discipline, where the tendrils of our craft are intertwined with nearly every other field of study. Because each discipline and each topic must be taught somehow, educational technologists exist at the crossroads to assist in designing the learning environments, instructional strategies, and technologies for teaching and learning. In collaborating with various disciplines, we use theoretical frameworks to explain our own understandings, as well as to develop common understanding at these intersections of knowledge. As such, we must be able to articulate what we know for our collaborating partners on interdisciplinary teams, as well as to develop new understanding for what we can accomplish together.

For these reasons, our field has always required theoretical work that can provide the foundations for both scientific advancement and the development of robust interventions in practice. We are well aware that our discipline has a rich history of theoretical scholarship, as we have drawn insights from cognitive psychology, computer science, communication studies, sociology, social psychology, educational psychology, human development, and other sciences of learning and instruction, and debated their merits within Educational Technology Research and Development, alongside other journals. In fact, many of the most highly cited papers in our discipline have been conceptual in nature. West and Borup (2014) analyzed ten years of scholarship in 10 top journals in the field of instructional technology, and found that 7 of the top 9 most highly cited papers were conceptual

Susan McKenney

susan.mckenney@utwente.nl

Richard E. West

rickwest@byu.edu

1 Brigham Young University, Provo, USA

2 Purdue University, West Lafayette, USA

3 University of Twente, Enschede, The Netherlands 
(theoretical or literature-based) rather than empirical. Further, when West analyzed a decade of research trends in over 20 journals related to our field, he concluded, "we consistently found theoretical/literature-based articles to be among the most cited in a journal" (West 2016, p. 44).

However, with the increasing emphasis on data-driven decision-making, evidence-based research, and empiricism, the value of theoretical scholarship risks being overlooked. Increasingly, journal editors appear to privilege empirical work at the cost of theoretical and conceptual scholarship. Sometimes this has been explicit, as outlined in the aims and scope of the journal, but other times it has been implicit, for example by using review criteria that align with the elements of empirical papers only. This is problematic, as all social science fields, including our own, depend on theoretical scholarship to inform both research and practice.

In order to embrace and showcase the crucial role that theoretical scholarship plays in our field, this special issue of Educational Technology Research and Development was designed to celebrate the long-standing tradition in the journal, and in the field, of encouraging rich, critical, and thought-provoking theoretical scholarship. As Whetten (1989) explained, such scholarship not only identifies the variables involved in a research agenda, but also explains, contextualizes, and critically analyzes the relationships between these variables. We need this kind of theoretical work to provoke robust empirical scholarship and guide quality design and practice.

\section{What is sound theoretical scholarship?}

To advance the field, our theoretical scholarship must be more than simply reflective practice or editorializing opinions. We must be able to demonstrate how this kind of work is sufficiently rigorous to nourish research and practice. But what are the qualities of rigorous theoretical scholarship?

Corley and Gioia (2011) synthesized arguments about the theoretical contributions in the field of business management which, like our field, "self-identif[ies] as 'borrowers' from many other scientific disciplines (e.g., psychology, sociology, economics, etc.) but ... claim[s] to speak to both academics and practitioners" (p. 12). The authors delineated two key dimensions of quality theoretical scholarship: originality and utility. First, theoretical work must be original; it must contribute new ideas to the academic discourse by providing original synthesis, applications to practice, methodological development, or wholly new conceptual understandings about the variables and influences related to teaching and learning. Second, theoretical scholarship must be useful, particularly in a discipline such as ours in which scholars see themselves as educational design researchers, seeking to influence 
local practice as much as create knowledge that can advance the work of others (McKenney and Reeves 2019). In our view, theory is important to the degree that it is useful and can have an actual impact on the world in which we live.

Whetten (1989) described several practical considerations for any scholarship, including theoretical scholarship: "Is the paper well written? Does it flow logically? Are the central ideas easily accessed? Is it enjoyable to read? Is the paper long enough to cover the subject but short enough to be interesting?" We have found that aspects such as these rarely appear in manuscript review guidelines, and that few journals adequately prepare reviewers to consider and/or apply criteria relevant to quality theoretical scholarship. While most journals provide standard rubrics for reviewers to complete, they generally focus on aspects such as writing clarity, formatting, and a strong justification of the need for the scholarship. Additionally, they usually require reviewers to evaluate the research design of the study being described, including data collection and analysis methods. These criteria are not relevant to theoretical scholarship, and might lead reviewers to assume that the journal does not welcome theoretical work. Without other guidance, reviewers may provide low evaluation scores, including comments such as, "this paper does not provide empirical research, and thus cannot be published."

Our hope is that this special issue will spark a dialogue among the field's scholars regarding theoretical contributions, including a discussion of what counts as relevant criteria for reviewing these types of papers. To begin that conversation, we created a list of evaluative criteria, inspired by the earlier criteria of originality, utility, and communicative ability (Corley and Gioia 2011), as well as selected aspects mentioned by Whetten (1989). While others have suggested different words or definitions for their criteria (and that conversation is important to continue!), we offered the following rubric to the reviewers of this special issue, asking them to rate each paper on a scale of 1 to 3 for each: 
The article should be...

INSTRUCTIVE - The article and its theoretical framework expands our current perspective and makes a genuine contribution to the field.

$1=$ The issues, concerns, or questions seem to be inappropriate, unimportant, or do not match needs identified in the literature. The questions or purposes are vague, too broad, or too narrow in scope. There is a poor connection between the rationale and the specific issues or thesis being defended.

$2=$ The issues, concerns, or questions are not as narrow in scope as they should be, not articulated well, or not strongly contextualized in the literature. The connection between the rationale for the scholarship, the paper's purpose, and the specific issues or thesis being defended is weak.

$3=$ The issues, concerns, or questions are clearly identified and justified. The purpose is clearly articulated and persuasively argued as important to the discipline. The connection between the rationale for the research, the paper's purpose, and the issues or thesis is clear.

SCHOLARLY - The article and its theoretical framework suggest new research and/or development directions for addressing urgent issues/problems.

$1=$ The article does not effectively articulate new research and/or development directions, nor are these suggested by the theoretical framework.

$2=$ The article and its theoretical framework suggest some research and/or theoretical directions, but they are either insufficiently clarified or persuasively argued as important.

$3=$ The article and its theoretical framework clearly identify and justify research and/or theoretical directions, which are persuasively argued as important to the discipline.

PRACTICAL - The article and its theoretical framework suggest new recommendations for Learning, Design, and Technology practice.

$1=$ The article does not effectively articulate implications for practice.

$2=$ The article and its theoretical framework suggest some practical implications, but they are either unimportant, or insufficiently linked to the theoretical framework. 
$3=$ Practical issues, concerns, or implications are clearly identified and developed from the theoretical framework, and explained in a way to further work in the discipline.

LOGICAL - The article provides a strong, logical argument, using existing literature or evidence.

$1=$ Argumentation is weak and includes invalid logical form, fallacies, unsupported claims, or gaps in reasoning. The article lacks coherent organization and clear focus. It fails to position the argument within a theoretical tradition, address rival positions or clarify important assumptions.

2=Argumentation needs strengthening, as it is not always clear, strong, and logically valid. Areas to improve include one or more of the following: Support for claims; positioning within a theoretical tradition; discussion of rival positions; organization, structure, or flow; gaps in reasoning.

$3=$ Argumentation is clear, strong, and logically valid. Claims are well supported. Conclusions clearly follow from prior theory, evidence, or information. The argument has effective organization and flow, and there are no obvious gaps in the reasoning. Important assumptions and rival positions are discussed, and situated within a theoretical tradition.

WRITING QUALITY - The article has clarity of expression, flow, and effective use of headings and visuals.

1- The article has many flaws with regard to mechanics, layout, or appearance that detract significantly from the readability. Writing is awkward, unclear, or unprofessional. Key guidelines have only partially been followed, e.g.violations of APA style.

2- The article has some flaws that affect the readability. These pertain to one or more of the following: mechanics, layout, and appearance; clear and professional style; APA formatting guidelines.

3- The article is professionally presented with regard to mechanics, layout, and appearance. Writing is clear and professional. APA formatting guidelines have been followed. Errors are few and do not detract from the overall quality of the writing. 
We do not argue that this is the perfect set of criteria for evaluating theoretical scholarship, but believe it offers a useful start to shifting the culture around, and developing more shared understanding about, theoretical contributions to our journals. When current rubrics are biased (e.g. toward empirical articles), we encourage journal editors, editorial boards, and reviewers to consider these and other appropriate criteria for evaluating theoretical submissions.

We also, as a field, need to expand this conversation to consider what the appropriate criteria would be for reviewing literature reviews, or design cases, or other types of useful scholarship that are not empirical. Ideally, journals would have unique sets of criteria for each type of submission they are open to receiving. As a global discipline, with new colleagues joining our reviewing ranks every year, there is an important need for clear norms around quality and valuable scholarship.

\section{Contributions in this special issue}

Judging by the nearly 100 proposals that were submitted to this special issue, we conclude that its focus resonated with many scholars. The selection process was extremely difficult. Based on the feedback received from reviewers using the above criteria, we are pleased to share a strong collection of articles which, together, demonstrate the rich diversity of theoretical work in our discipline. We begin the special issue with two papers describing how to develop quality theory in our field. Melissa Warr, Punya Mishra, and Benjamin Scragg begin by asking us as a discipline to apply the same design thinking and problem solving that we use in creating educational design to instead create theory. They argue that by doing so, our theory can better bridge the gap between research and practice, explain solutions to "wickedly" complex problems, and embed theory development into regular practice. Similarly, Jason McDonald and Stephen Yanchar argue in their paper that our field should focus on developing originary theory, or theory generated by the scholars and practitioners intending to apply it. They contrast this with imported theory, brought from other domains, which is often misfitted. Together, these papers push our thinking as a discipline about what counts as theory, whose responsibility it is to develop theory, and how theory can feed our design practice as well as our scholarship.

The remainder of the articles in the special issue articulate different theoretical frameworks that can be applied to specific educational settings, or to specific instructional activities. Of these, two papers offer broadly applicable instructional design principles. Andrew Tawfik, Andrew Graesser, Jessica Gatewood, and Jaclyn Gishbaugher begin with a design taxonomy for instructional activities focused on questioning and guiding student inquiry. Similar to inquiry-based education, Krista Glazewski and Peg Ertmer discuss complex problem solving, and encourage us to consider principles for how to apply problem-based learning to foster greater equity and social justice.

Following these two papers, we turn to the domain of teacher learning and practice. Sara van der Linden and Susan McKenney argue for uniting cognitive and situated paradigms of teacher knowledge development, and discuss the related implications for coaching teachers with the aid of video. In the next paper, Theodore Kopcha, Kalianne Neumann, Anne Ottenbreit-Leftwich, and Elizabeth Pitman argue for an evolution in our understanding of teacher technological use, away from a focus on the products that teachers create and instead towards emphasizing principles for understanding contextualized teacher decision making about technology integration. 
The final three papers in the special issue present theoretical frameworks for guiding our online teaching practices. Daniel Hickey, Grant Chartrand, and Christopher Andrews analyze theoretical perspectives on the framing of instruction, including how, when, and by whom this framing should be done for maximum learning benefit. They provide examples in the context of online learning, although their ideas would have applicability in other contexts as well. Jeroen Janssen and Paul Kirschner elaborate on traditional cognitive load theory in light of computer-supported collaborative learning environments, presenting robust ideas for research and practice. Finally, Jered Borup, Charles Graham, Richard West, Leanna Archambault, and Kristian Spring share a communities of engagement framework: a collection of ideas and principles that explain how students engage affectively, behaviorally, and cognitively in online learning environments; and describe the roles that various actors within students' personal and course communities play in increasing students' engagement and learning.

\section{Conclusion and call for continued theoretical work}

The papers in this special issue comprise important contributions to their individual domains of research and practice. Beyond their individual contributions, the collection of papers presented in this special issue contributes to the evolution of the field by providing examples of and arguments for the future of theoretical scholarship. With continued technological advances, globalization of our social institutions and societies, and the complexity of twentyfirst century challenges, we will continue to look to evolving theory to guide our discipline. In order to meet these opportunities, our field needs to continue the conversation about how, and what kinds of, theoretical contributions can guide us. This special issue (and especially the rubric provided above) offers important considerations related to the rigor and quality of theoretical scholarship. It demonstrates how we can promote and develop a more supportive culture for this type of work. Further scholarship is needed to adequately address questions like: How do we best support students and new scholars to do theoretical work? What are the next areas in which theoretical work is needed to advance the field of educational technology?

Similarly to how we emphasize research methods and design processes in our academies, we need to better understand the skills, criteria, and roles of quality theoretical scholarship. We hope this special issue fosters a continuation of this dialogue.

Open Access This article is licensed under a Creative Commons Attribution 4.0 International License, which permits use, sharing, adaptation, distribution and reproduction in any medium or format, as long as you give appropriate credit to the original author(s) and the source, provide a link to the Creative Commons licence, and indicate if changes were made. The images or other third party material in this article are included in the article's Creative Commons licence, unless indicated otherwise in a credit line to the material. If material is not included in the article's Creative Commons licence and your intended use is not permitted by statutory regulation or exceeds the permitted use, you will need to obtain permission directly from the copyright holder. To view a copy of this licence, visit http://creativecommons.org/licenses/by/4.0/.

\section{References}

Corley, K., \& Gioia, D. (2011). Building theory about theory building: What constitutes a theoretical contribution? Academy of Management Review, 36(1), 12-32. https://doi.org/10.5465/amr.2009.0486.

McKenney, S., \& Reeves, T. C. (2019). Conducting educational design research (2nd Ed.). New York, NY: Routledge.

West, R. E. (2016). Insights from the journal analysis series: What we have learned about educational technology research. Educational Technology, 56, 41-45. 
West, R. E., \& Borup, J. (2014). An analysis of a decade of research in 10 instructional design and technology journals. British Journal of Educational Technology, 45, 545-556. https://doi.org/10.1111/bjet.12081.

Whetten, D. A. (1989). What constitutes a theoretical contribution? Academy of Management Review, 14, 490-495.

Publisher's Note Springer Nature remains neutral with regard to jurisdictional claims in published maps and institutional affiliations.

Richard E. West is an associate professor in the Instructional Psychology and Technology department at Brigham Young University. He teaches courses in instructional technology, academic research and writing, creativity and innovation, and product/program evaluation. He researches how to create learning environments that prepare students for the 21 st century, including designing learning environments that foster group creativity through design thinking, utilizing microcredentials and open badges to promote competency learning, the development and support of online learning communities, and emerging technologies to support effective learning.

Peg Ertmer is professor emerita in the Learning Design and Technology program in the College of Education at Purdue University. Her research examines the impact that student-centered instructional approaches, including case- and problem-based learning, have on learning.

Susan McKenney is professor of teacher professionalization, school development and educational technology at the University of Twente in the Netherlands. Her research focuses on these three themes, often in relation to curriculum design, and frequently featuring synergistic research-practice interactions. 